\section{Acknowledgments}

The authors' research into the role of filaggrin in the genetics of atopic eczema is funded by Wellcome Trust Programme (092530/Z/10/Z; to W.H.I. McLean and A.D. Irvine), and Bioresources grants (090066/B/09/Z; to A.D. Irvine and W.H.I. McLean). The Centre for Dermatology and Genetic Medicine, University of Dundee is supported by a Wellcome Trust Strategic Award (098439/Z/12/Z; to W.H.I. McLean).

Address correspondence to: W.H. Irwin McLean, Centre for Dermatology and Genetic Medicine, Division of Molecular Medicine, Colleges of Life Sciences and Medicine, Dentistry and Nursing, University of Dundee, Dow Street, Dundee DD1 5EH, United Kingdom. Phone: 44.1382.381046; Fax: 44.1382.388535; E-mail: w.h.i.mclean@dundee.ac.uk.
1. Irvine AD, McLean WHI, Leung DY. Filaggrin mutations associated with skin and allergic diseases. N Engl J Med. 2011;365(14):1315-1327.

2. Novak N, Leung DY. Advances in atopic dermatitis. Curr Opin Immunol. 2011;23(6):778-783.

3. Smith FJD, et al. Loss-of-function mutations in the gene encoding filaggrin cause ichthyosis vulgaris. Nat Genet. 2006;38(3):337-342.

4. Sandilands A, et al. Comprehensive analysis of the gene encoding filaggrin uncovers prevalent and rare mutations in ichthyosis vulgaris and atopic eczema. Nat Genet. 2007;39(5):650-654.

5. Palmer CNA, et al. Common loss-of-function variants of the epidermal barrier protein filaggrin are a major predisposing factor for atopic dermatitis. Nat Genet. 2006;38(4):441-446.

6. Fallon PG, et al. A homozygous frameshift mutation in the mouse Flg gene facilitates enhanced percutaneous allergen priming. Nat Genet. 2009;41(5):602-608.

7. Dioscorides P. Book 1: Aromatics. In: Osbaldeston TA, Wood RPA, eds. Dioscorides: De Materia Medica (A new indexed version in modern English). Johannesburg, South Africa: Ibidis Press; 2000:99-100.

8. Thami GP, Sarkar R. Coal tar: past, present and future. Clin Exp Dermatol. 2002;27(2):99-103

9. Slutsky JB, Clark RA, Remedios AA, Klein PA.
An evidence-based review of the efficacy of coal tar preparations in the treatment of psoriasis and atopic dermatitis. J Drugs Dermatol. 2010; 9(10):1258-1264.

10. Mainstone J. The Pitch Drop Experiment. The University of Queensland Web site. http://smp.uq.edu. $\mathrm{au} /$ content/pitch-drop-experiment. Accessed December 10, 2012

11. Choudhary G, Citra MJ, McDonald AR, QuiñoneRivera A. Toxicological Profile for Wood Creosote, Coal Tar Creosote, Coal Tar, Coal Tar Pitch, and Coal Tar Pitch Volatiles. Atlanta, Georgia, USA; US Department of Health and Human Services; 2002.

12. van den Bogaard EH, et al. Coal tar induces AHRdependent skin barrier repair in atopic dermatitis. J Clin Invest. 2013;123(2):917-927.

13. Du L, Hoffman SM, Keeney DS. Epidermal CYP2 family cytochromes P450. Toxicol Appl Pharmacol. 2004;195(3):278-287.

14. Smith G, et al. Regulation of cutaneous drugmetabolizing enzymes and cytoprotective gene expression by topical drugs in human skin in vivo. Br J Dermatol. 2006;155(2):275-281.

15. Roelofzen JH, et al. DNA adducts in skin biopsies and 1-hydroxypyrene in urine of psoriasis patients and healthy volunteers following treatment with coal tar. Toxicol Lett. 2012;213(1):39-44.

\title{
Nucleocytoplasmic connections and deafness
}

\author{
Howard J. Worman ${ }^{1}$ and Neil Segil2,3
}

1Departments of Medicine and of Pathology and Cell Biology, College of Physicians and Surgeons, Columbia University, New York, New York, USA. 2Division of Cell Biology and Genetics, House Research Institute and ${ }^{3}$ Department of Cell and Neurobiology, Keck School of Medicine, University of Southern California, Los Angeles, California, USA.

\begin{abstract}
The linker of nucleoskeleton and cytoskeleton (LINC) complex connects the nuclear lamina to the cytoskeleton, in part to aid in nuclear positioning. Mutations in genes encoding LINC complex and lamina components cause a range of human diseases. In this issue of the JCI, Horn et al. report that mutations in the gene SYNE4 encoding the LINC complex protein nesprin-4 lead to progressive high-frequency hearing loss. Further, in mice deficient in nesprin-4 and Sun1, another LINC complex component, outer hair cells of the cochlea form normally during development, but die in the early postnatal weeks. These results link improper nuclear positioning specifically to the death of outer hair cells in the organ of Corti and ultimately to deafness.
\end{abstract}

The nuclear envelope is composed of the nuclear membranes (inner and outer), nuclear pore complexes, and nuclear lamina. It separates the nucleoplasm from the cytoplasm of eukaryotic cells, and the transport of proteins, nucleic acids, and other molecules among these compartments in interphase is restricted to the pore complexes. The nuclear envelope has been a growing focus of

Conflict of interest: The authors have declared that no conflict of interest exists.

Citation for this article: J Clin Invest. 2013; 123(2):553-555. doi:10.1172/JCI67454. clinical investigation, as in the past 15 years a wide range of inherited diseases have been linked to mutations in genes encoding proteins of this subcellular structure $(1,2)$.

\section{Nuclear envelope and nucleocytoplasmic connections}

Recent research has shown that the nuclear envelope not only separates the nucleus from the cytoplasm, but also connects the structural networks of these subcellular compartments (Figure 1). The linker of nucleoskeleton and cytoskeleton (LINC) complex mediates this con- nection (3). The core of the LINC complex forms from the interaction of SUN (Sad1, UNC-84) domain proteins with KASH (Klarsicht, ANC-1, Syne homology) domain proteins called nesprins (4, 5 ). This creates a protein bridge spanning the inner and outer nuclear membranes. The SUN domains, attached to a trimeric coiled coil of Sun proteins, bind three KASH domains, with a disulfide bond between cysteines in SUN and KASH further covalently linking them (6). Suns are integral proteins of the inner nuclear membrane that also bind to A-type lamins of the nuclear lamina, a meshwork of intermediate filament proteins providing structural support to the nucleus. The SUN-KASH binding within the perinuclear space retains the nesprin, which also contains a transmembrane segment, in the outer nuclear membrane. At the other end of this transnuclear membrane bridge, different nesprins interact with unique cytoskeletal components. For example, nesprin-1 and nesprin-2 isoforms bind directly to actin, nesprin-3 via plectin to cytoplasmic intermediate 


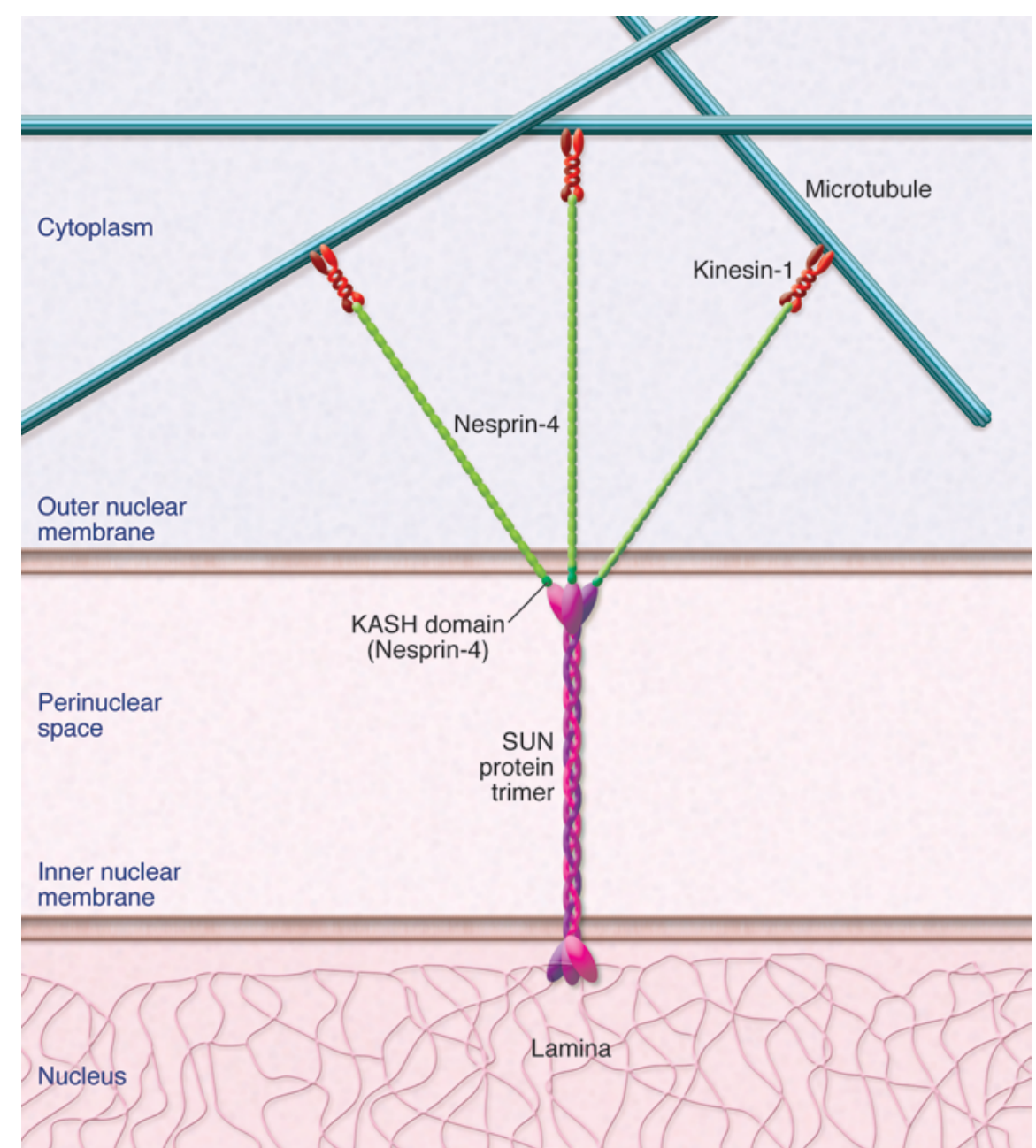

filaments, and nesprin-4 to kinesin (4, 5 ). As a result of these series of interactions, the nucleoskeleton is connected to the cytoskeleton.

The nucleocytoplasmic interactions established by the LINC complex play an important role in the transmission of mechanical forces across the nuclear envelope and in nuclear movement and positioning. In migrating fibroblasts, linear arrays of nesprin-2-G and Sun2 mediate the retrograde actin-dependent movement of nuclei (7). In mammalian neurons, Sun $1 / 2$ and nesprin- $1 / 2$ function in microtubule-dependent nuclear migration during neurogenesis via their interactions with dynein and kinesin-1 (8). Nesprin-4 binds to kinesin, and its expression in cultured cells is associated with changes in the locations of the centrosome and Golgi apparatus relative to the nucleus, suggesting a role in microtubule-dependent nuclear positioning (9). The relevance of these nuclear position- ing processes to health and disease are only beginning to be understood.

\section{Linking the LINC complex to deafness}

In this issue of the JCI, Horn et al. report that mutations in the gene SYNE4, encoding nesprin-4, cause autosomal recessive, progressive high frequency hearing loss in two families of Iraqi Jewish ancestry (10). Nesprin-4 was previously shown to be present in the nuclear envelope in cells of the salivary gland, exocrine pancreas, bulbourethral gland, mammary gland, and in some kidney cells (9), among others. In the current work, Horn et al. show its expression in hair cells of the cochlea, specifically the three rows of outer hair cells and one row of inner hair cells in the organ of Corti. Further, Horn et al. demonstrated that nesprin-4-deficient mice - as well as mice lacking Sun 1 - have progressive hearing loss. In these mice, outer hair

\section{Figure 1}

The LINC complex and its connection to the nuclear lamina and cytoskeletal components. The carboxyl termini of Suns, integral proteins of the inner nuclear membrane, bind within the perinuclear space to the carboxyl terminal KASH domain of nesprins, which are transmembrane proteins localized to the outer nuclear membrane. The amino termini of Suns bind to the nuclear lamina, an intermediate filament meshwork of lamins on the inner aspect of the inner nuclear membrane. Different nesprins have variable cytoplasmic amino terminal domains that interact with different cytoskeletal elements. Nesprin-4, shown in this diagram, binds to kinesin, which in turn can associate with microtubules. cells are formed but appear to degenerate as hearing matures, while the inner hair cells remain intact. Nuclei of the outer hair cells fail to maintain a normal basal localization. The authors conclude that the nucleocytoplasmic connections established by the LINC complex are essential for viability of the outer hair cells and propose that proper nuclear positioning in these cells is critical to the maintenance of normal hearing (10).

\section{Perspective and remaining questions} Sensory hair cells in the mammalian inner ear are extremely sensitive to environmental stress and undergo apoptosis in response. While hair cells in nonmammalian vertebrates are able to regenerate from the surrounding supporting cells, mammalian hair cells do not, so their degeneration leads to permanent hearing loss (11). The basal-to-apical gradient of cell death Horn et al. observed in mice with defects in the LINC complex is 
common to many forms of cochlear damage, including acoustic trauma and exposure to aminoglycoside antibiotics and chemotherapy agents (12). The underlying causes of this gradient of sensitivity are poorly understood but have been hypothesized to result from a gradient in stress-induced sensitivity to reactive oxygen species or apoptotic signaling. It will therefore be interesting to test whether therapies that mitigate the normal sensitivity to stress in these cells, such as treatment with free radical scavengers, also mitigate the precocious outer hair cell degeneration in the nesprin-4-deficient and Sun1-deficient mice.

Nesprin-4 and Sun 1 are present in the nuclear envelopes of both inner and outer hair cells of the organ of Corti; however, only outer hair cells appear to be severely affected by deletion of their genes. Horn et al. hypothesize that this is the result of extreme mechanical stress placed on these cells because of their wellknown power of movement in response to sound as part of the cochlear amplifier (10). However, depletion of Suns and nesprin-2 has been shown to lead to a very similar degeneration of postmitotic photoreceptors in the mouse eye (13). This suggests that other processes may be at work, since photoreceptors do not harbor the same properties of electromotility as outer hair cells. It is therefore important to directly determine whether the electromotility of outer hair cells is indeed affected in nesprin-4-deficient and Sun1-deficient mice, as hypothesized, and which apoptotic pathways may be activated as a result of any observed abnormality. In addition to sparing of the inner hair cells, it is also unclear why organs other than the inner ear containing cells that express nesprin-4 and Sun 1 and may be subjected to various stressful forces are apparently not affected in the knockout mice.

Thus, while the effects of LINC complex defects on the unique electromechanical properties of outer hair cells of the inner ear is a parsimonious hypothesis, alternative mechanisms need to be ruled out. Alterations in nesprins and Sun proteins could potentially affect cellular functions in ways that do not result from the altered transmission of mechan- ical forces across the nuclear envelope or abnormal nuclear positioning. For instance, Sun proteins interact directly and indirectly with several elements of the nuclear interior, including chromatin (14). Hence, mutations in their genes could lead to changes in cell type-specific gene expression that are detrimental to physiological function and result from abnormalities in nuclear functions separate from those involving connecting the nucleoskeleton to the cytoskeleton.

Finally, mutations in genes encoding proteins of the LINC complex and associated structures cause many disorders other than hearing loss. Mutations in SYNE1, encoding nesprin-1, cause an autosomal recessive cerebellar ataxia (15). They have also been reported to cause an autosomal recessive form of arthrogryposis multiplex congenita characterized by decreased fetal movements, delay in motor milestones, and progressive motor decline after the first decade (16). SYNE1 and SYNE2 mutations have further been implicated in Emery-Dreifuss muscular dystrophy-like phenotypes $(17,18)$. Various mutations in the lamin A/C gene (LMNA) cause several different tissue-selective diseases $(1,2)$, but an effect on nuclear movement has only been shown for a subset of LMNA mutations that cause cardiomyopathy and muscular dystrophy and only in a model fibroblast system (19). These fascinating results point to the need for a better understanding of the tissue- and cell-selective expression of the protein components of the LINC complex and associated structures. In addition, a better understanding of the functional roles of each of these proteins in force transmission across the nuclear envelope and in nuclear movement and positioning is required to explain the divergent phenotypes of diseases caused by alterations in nucleocytoplasmic connections.

\section{Acknowledgments}

H.J. Worman is supported by NIH grants NS059352 and HD070713 for research related to this commentary.

Address correspondence to: Howard J. Worman, Department of Medicine, College of Physician and Surgeons, Colum- bia University, 630 West 168th Street, New York, New York 10032, USA. Phone: 212.305.1306; Fax: 212.342.0509; E-mail: hjw14@columbia.edu.

1. Worman HJ, Fong LG, Muchir A, Young SG. Laminopathies and the long strange trip from basic cell biology to therapy. J Clin Invest. 2009; 119(7):1825-1836.

2. Dauer WT, Worman HJ. The nuclear envelope as a signaling node in development and disease. Dev Cell. 2009;17(5):626-638.

3. Crisp $M$, et al. Coupling of the nucleus and cytoplasm: role of the LINC complex. J Cell Biol. 2006;172(1):41-53.

4. Worman HJ, Gundersen GG. Here come the SUNs: a nucleocytoskeletal missing link. Trends Cell Biol. 2006;16(2):67-69.

5. Starr DA. KASH and SUN proteins. Curr Biol. 2011;21(11):R414-R415.

6. Sosa BA, Rothballer A, Kutay U, Schwartz TU. LINC complexes form by binding of three KASH peptides to domain interfaces of trimeric SUN proteins. Cell. 2012;149(5):1035-1047.

7. Luxton GW, Gomes ER, Folker ES, Vintinner E, Gundersen GG. Linear arrays of nuclear envelope proteins harness retrograde actin flow for nuclear movement. Science. 2010;329(5994):956-959.

8. Zhang $X$, et al. SUN1/2 and Syne/Nesprin-1/2 complexes connect centrosome to the nucleus during neurogenesis and neuronal migration in mice. Neuron. 2009;64(2):173-187.

9. Roux KJ, et al. Nesprin 4 is an outer nuclear membrane protein that can induce kinesin-mediated cell polarization. Proc Natl Acad Sci U S A. 2009;106(7):2194-2199.

10. Horn HF, et al. The LINC complex is essential for hearing. J Clin Invest. 2013;123(2):740-750.

11. Kwan T, White PM, Segil N. Development and regeneration of the inner ear. Ann N Y Acad Sci. 2009;1170:28-33.

12. Op de Beeck K, Schacht J, Van Camp G. Apoptosis in acquired and genetic hearing impairment: the programmed death of the hair cell. Hear Res. 2011;281(1-2):18-27.

13. Yu J, et al. KASH protein Syne-2/Nesprin-2 and SUN proteins SUN1/2 mediate nuclear migration during mammalian retinal development. Hum Mol Genet. 2011;20(6):1061-1073.

14. Lombardi ML, Lammerding J. Keeping the LINC: the importance of nucleocytoskeletal coupling in intracellular force transmission and cellular function. Biochem Soc Trans. 2011;39(6):1729-1734.

15. Gros-Louis F, et al. Mutations in SYNE1 lead to a newly discovered form of autosomal recessive cerebellar ataxia. Nat Genet. 2007;39(1):80-85.

16. Attali R, et al. Mutation of SYNE-1, encoding an essential component of the nuclear lamina, is responsible for autosomal recessive arthrogryposis. Hum Mol Genet. 2009;18(18):3462-3469.

17. Zhang $Q$, et al. Nesprin- 1 and -2 are involved in the pathogenesis of Emery Dreifuss muscular dystrophy and are critical for nuclear envelope integrity. Hum Mol Genet. 2007;16(23):2816-2833.

18. Puckelwartz MJ, et al. Nesprin-1 mutations in human and murine cardiomyopathy. J Mol Cell Cardiol. 2010;48(4):600-608.

19. Folker ES, Östlund C, Luxton GW, Worman HJ, Gundersen GG. Lamin A variants that cause striated muscle disease are defective in anchoring transmembrane actin-associated nuclear lines for nuclear movement. Proc Natl Acad Sci U S A. 2011;108(1):131-136. 